\title{
Context Engineering Hybrid Spaces for Perceptual Augmentation
}

\author{
Carl H. Smith \\ Learning Technology Research Institute (LTRI) \\ London Metropolitan University \\ London, United Kingdom \\ carl.smith@londonmet.ac.uk
}

\section{INTRODUCTION}

This paper is concerned with examining how emerging spatial technologies are reconfiguring perception. The dominant perceptive regimen is still based on the Cartesian split (subject-object separation). We see matter as separated from mind, a perceptive model inherited from literacyCartesian culture that has not yet digested the new changes brought about by hybrid techniques and methodologies. This dominance is also reflected in the way we deal with the consensus construction of reality alongside our definitions of what consciousness is and can be. The role that hybrid space plays in the construction and transformation of perception through the practise of context engineering will be examined. Context engineering is understood as an "intermediality" practice for exploring perceptual augmentation by giving us control over our senses, allowing us to adjust them in real time.

\section{AUGMENTING PERSPECTIVE}

Perspective has become such a powerful and pervasive paradigm that it is difficult to imagine perceiving the world without it. At the same time, its effects on human consciousness are so subtle and insidious that one is rarely aware of it.... The perceptual technologies by which forms are configured within a culture mediate certain patterns of association that affect the perceptual disposition of that culture. It follows that when perceptual technologies change, the perceptual disposition of the culture may also undergo a transformation. (Shanken 1996).

The eye now adjusts so readily to the effects of linear perspective that it is considered to be an innate process. The evolution of our perception alongside our architectural understanding is dictated by spatial conventions that have been built into our cultural constructs since the Renaissance. Panofsky (1924) in his book 'Perspective as a Symbolic Form' established a parallel between the history of spatial representation and the evolution of abstract thought.

Vision and optical experts (Radley 2008) note that it is rather curvilinear perspective that has a strong foundation in reality as the human visual field has a natural curvilinear shape. As a result abstract reasoning which is developed through the use of media tools and media content that are constructed using earlier conventions can now be augmented with other more 'realistic' forms of spatial construction.

\section{HYBRID PERCEPTION}

When the digital blends with the physical, in particular in relation to spatial perception, a hybrid reality appears and the wide and varied impact on perception now requires mapping.

Firstly, I will focus on how hybrid space creates an opportunity to perform context engineering (Smith 2013) by maximising our spatial interaction with the environment, going beyond the traditional emphasis on surface, content and representation (Ascott 2002). Secondly, I will analyse how the design and implementation of hybrid spaces can transform perception.

The analysis will be carried out through a number of case studies investigating field of view (FOV) technologies and new forms of macroscopic navigation. The FlyVIZ headset augments the sense of sight by giving the user 360 -degree vision. The 360 view is compressed to fit into a human's usual 180 degree field of view, it takes fifteen minutes for the brain to adjust to this new way of seeing and 'accept it as normal' (Ardouin et al. 2012). The ability within one field of view, to be 
both in the world and to see yourself in it, the power of looking through, and occupying, your own field of vision (Webb 2009) is becoming a hybrid tool for perception adaptation.

The results of this analysis will then be synthesised in a comprehensive overview of the possible new ways in which perception and consciousness can be transformed through the process of context engineering. The impacts on art and learning science will be highlighted.

\section{REFERENCES}

Ardouin, J., Lécuyer, A., Marchal, M., Riant, C. and Marchand, E. (2012) FlyVIZ: a novel display device to provide humans with $360^{\circ}$ vision by coupling catadioptric camera with HDM. In Proceedings of the 18th ACM Symposium on Virtual Reality Software and Technology, pp. 41-44.

Ascott, R. (2002) The architecture of cyberception. Architects in cyberspace. Architectural Design Profile N, 18, 38-41.
Panofsky, E. (1927) Die perspektive als symbolische form. In Vorträge der Bibliothek Warburg, Leipzig, Berlin, 1924-25.

Radley, A. (2008) The Lookable User Interface and 3D. $\quad$ https://sites.google.com/site/alanradley3/ publications (retrieved 18 May 2014).

Shanken, E. A. (1996) Virtual perspective and the artistic vision: a genealogy of technology, perception, and power. In Proc. ISEA96, pp. 57-63.

Smith, C. (2013) (Re)engineering cultural heritage contexts using creative human computer interaction techniques and mixed reality methodologies. In F. V. Cipolla-Ficarra (ed.), Advanced Research and Trends in New Technologies, Software, HumanComputer Interaction, and Communicability, IGI Global, Chapter 40, pp. 441-451.

Webb, M. (2009) Maps and macroscopes. Scroll Magazine. http://berglondon.com/blog/2010/02/03/ maps-and-macroscopes/ (retrieved 18 May 2014). 